\title{
HIGHLIGHTS
}

MOLECULAR IMAGING

\section{New CMR approach to measure diffuse myocardial fibrosis}

Cardiovascular magnetic resonance imaging (CMR) with late gadolinium enhancement can localize focal scar tissue with high, transmural resolution. However, this technique cannot detect diffuse myocardial fibrosis, which can only be assessed invasively by biopsy. James Moon and colleagues developed a new imaging approach-equilibrium contrast CMR (EQ-CMR) - that can quantify diffuse fibrosis noninvasively in humans.

\section{The correlation of EQ-CMR} signal with fibrosis assessed by biopsy was strong in patients with aortic stenosis... 77

Diffuse myocardial fibrosis "is the gradual thickening of the supportive connective tissue surrounding myocytes," explains Dr Moon, "a key process leading to diastolic and ultimately systolic dysfunction and breathlessness". This process is associated with ageing, but it is also increased in the setting of hypertension, aortic stenosis, or cardiomyopathy.

In CMR with late gadolinium enhancement-the current gold standard to assess myocardial fibrosis - the contrast agent is given in a single bolus. In scarred tissue, the contrast kinetics are slower than in healthy tissue, so the contrast agent washes out more slowly. In addition, fibrotic tissue has more free water accessible to contrast, so the signal is brighter. In EQ-CMR, however, a slow continuous infusion of the contrast agent is given after the initial bolus. "This makes concentrations in the blood and tissue equilibrate, so signal changes reflect the volume of distribution only and not the kinetics," says Dr Moon. The approach includes taking a blood sample to measure blood volume of distribution, from which the myocardial volume of distribution can be derived. By calibration with data from human biopsy samples, this measure can be converted to percentage of fibrosis.
The researchers validated the technique in 18 patients undergoing aortic valve replacement for aortic stenosis and 8 patients with hypertrophic cardiomyopathy undergoing myectomy. The correlation of EQ-CMR signal with fibrosis assessed by biopsy was strong in patients with aortic stenosis and in the total population of patients analyzed, and it was close to statistical significance in the cardiomyopathy group.

Moon and colleagues are now applying EQ-CMR in a range of cardiac conditions to see if diffuse fibrosis predicts important outcomes. Equilibrium contrast MRI can also potentially be used to measure diffuse fibrosis in any part of the body. "This promises to open up a whole new field of research," says Dr Moon.

Joana Osório

Original article Flett, A. S. Equilibrium contrast cardiovascular magnetic resonance for the measurement of diffuse myocardial fibrosis: preliminary validation in humans. Circulation 122, 138-144 (2010) 